\title{
THE NUMBER OF PARTITIONS OF A SET OF $N$ POINTS IN $k$ DIMENSIONS INDUCED BY HYPERPLANES
}

\author{
by E. F. HARDING $\dagger$ \\ (Received 21st October 1966)
}

1. An arbitrary $(k-1)$-dimensional hyperplane disconnects $k$-dimensional Euclidean space $E_{k}$ into two disjoint half-spaces. If a set of $N$ points in general position in $E_{k}$ is given [no $k+1$ in a $(k-1)$-plane, no $k$ in a $(k-2)$-plane, and so on], then the set is partitioned into two subsets by the hyperplane, a point belonging to one or the other subset according to which half-space it belongs to; for this purpose the half-spaces are considered as an unordered pair.

In some recent discussion on Cluster Analysis (a statistical activity in which methods are sought for partitioning a set of individuals, on the basis of their characteristics, into groups or clusters so that the members of a cluster are in some sense closely related) the following questions arose:

(i) What is the number, $v_{k}(N)$, of distinct partitions of a given set of $N$ points in $k$ dimensions that can be thus induced by $(k-1)$-dimensional hyperplanes? (Points are regarded as being distinguishably labelled, for instance by their coordinates).

(ii) How many such partitions remain if the partitioning plane is constrained to contain a given $j$-dimensional hyperplane $(j \leqq k-1)$ of $E_{k}$ that does not contain any of the given points?

In Figures $1 \mathrm{~A}$ and $1 \mathrm{~B}$ two different configurations of 4 points in $E_{2}$ are each partitioned by lines in 7 different ways. One may similarly verify that for 5 points there are 11 different partitions and that the result is independent of their configuration-though the number of partitions of a given type, say $(2,3)$, will be configuration-dependent.

From now on, "plane" will be used for "hyperplane" whenever no confusion will ensue.

2. The first question is answered by

Theorem 1. The number $v_{k}(N)$ defined in Question (i) is given by

$$
v_{k}(N)=\sum_{i=0}^{k}\left(\begin{array}{c}
N-1 \\
i
\end{array}\right)
$$

which for $N-1 \leqq k$ is interpreted as $2^{N-1}$.

$\dagger$ Much of this work was done while the author was at the University of Aberdeen. 


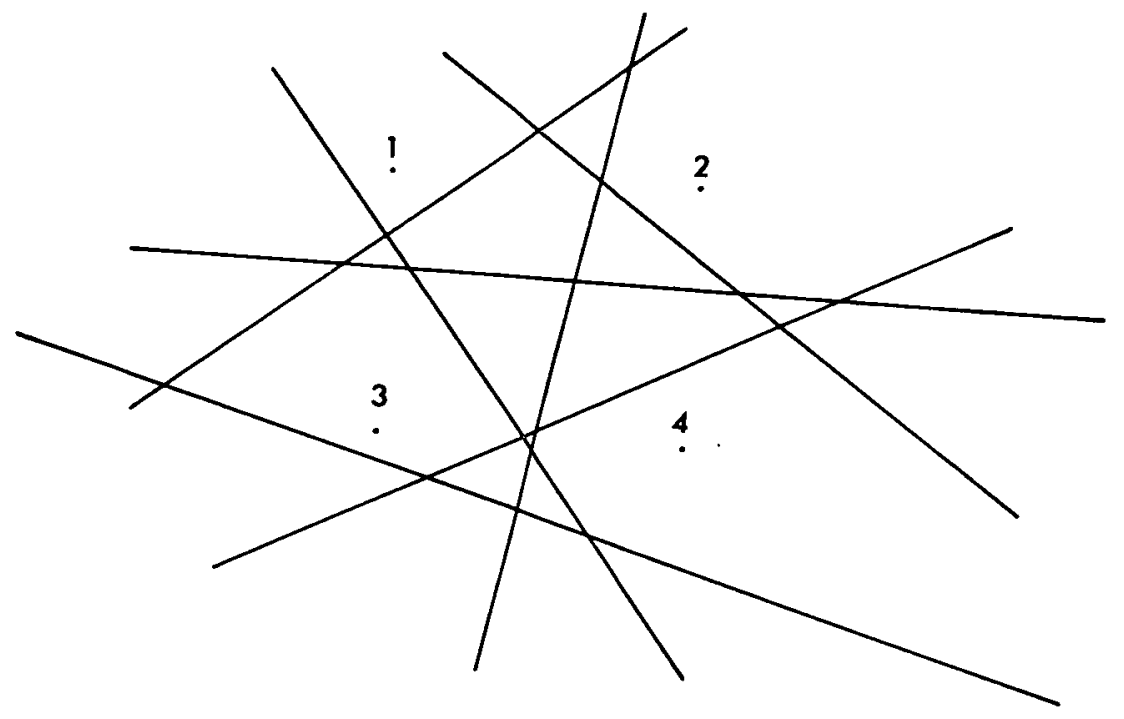

Fig. 1A

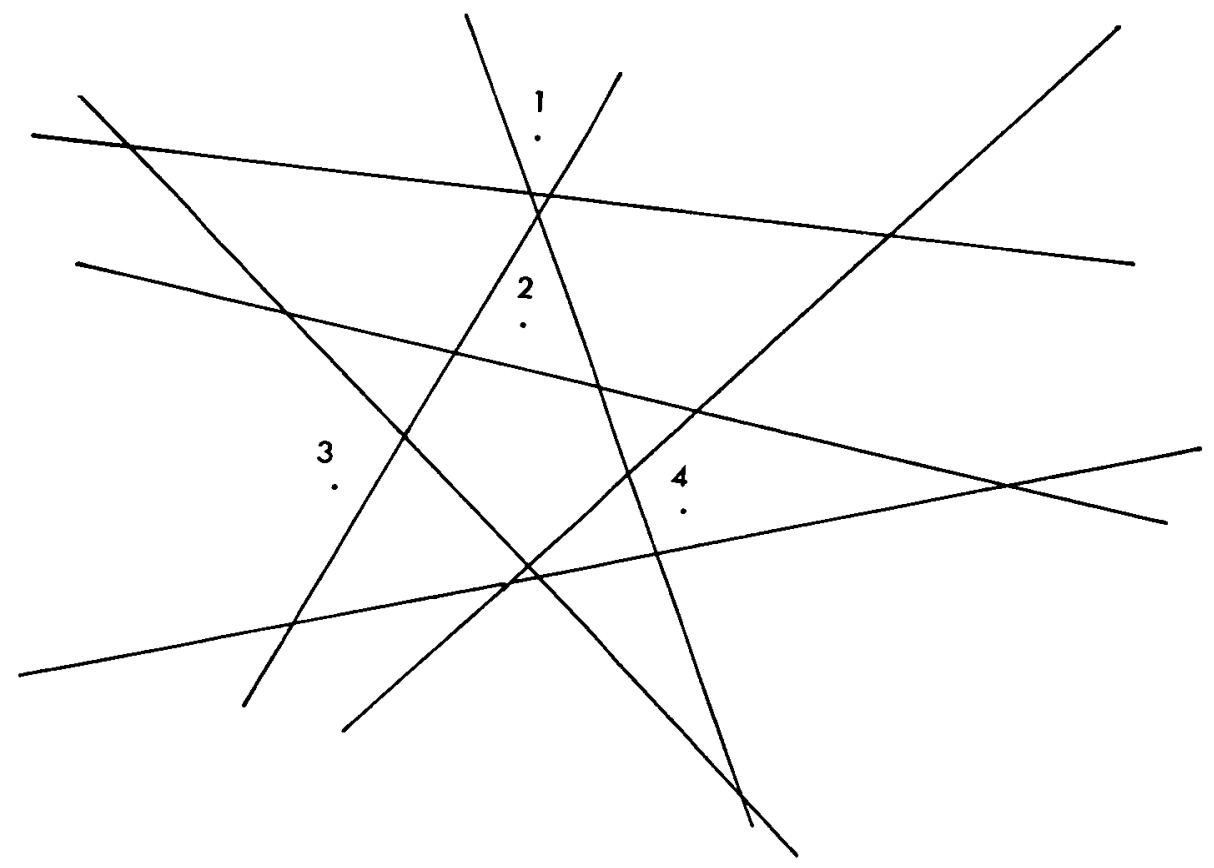

FIG, 1B 
Proof. Clearly

$$
v_{1}(N)=N \quad(N=1,2, \ldots) .
$$

We consider first the case $N-1 \geqq k$ and, proceeding by induction, show that for $k \geqq 2$

$$
v_{k}(N+1)=v_{k}(N)+v_{k-1}(N)
$$

Suppose $(A)$ true for numbers of points and dimensions up to and including $N$ and $k$ respectively. Let $C_{N+1}$ be any configuration of $N+1$ points in general position in $E_{k}$. Then at least 1 point, $P$ say, of $C_{N+1}$ may be separated by a $(k-1)$-plane, $\pi_{0}$ say, from the remainder which forms a configuration, $C_{N}$ say, to which $(A)$ applies. Thus $C_{N}$ can be partitioned in $v_{k}(N)$ ways by a set $[\pi]$ of $v_{k}(N)(k-1)$-planes $\pi$ of which none contain $P$ and one may be taken as $\pi_{0}$.

Now let the configuration $C_{N}$ be projected from $P$ onto the plane $\pi_{0}$ so as to form a configuration $C_{N}^{\prime}$ in general position in the $E_{k-1} \pi_{0} ; C_{N}^{\prime}$ can, by hypothesis, be partitioned in $v_{k-1}(N)$ ways by a set $\left[\pi^{*}\right]$ of $v_{k-1}(N)(k-1)$ planes $\pi^{*}$, each $\pi^{*}$ containing $P$ and meeting $\pi_{0}$ in a $(k-2)$-plane inducing the same partition of $C_{N}^{\prime}$ as the $\pi^{*}$. But there are also $v_{k-1}(N)$ planes of the set $[\pi]$ inducing the same partitions as do the set $\left[\pi^{*}\right]$, in one-to-one correspondence with the $\left[\pi^{*}\right]$. Let each $\pi^{*}$ be infinitesimally displaced away from $P$ without changing the partition induced in $C_{N}$, but so as to induce a different partition of $C_{N+1}$ from that induced by the corresponding $\pi$ : if $\pi$ induces the partition $\left(P, A_{1}, \ldots, A_{r} / B_{1}, \ldots, B_{N-r}\right)$ of $C_{N+1}$, the corresponding $\pi^{*}$ includes $P$ and induces the partition $\left(A_{1}, \ldots, A_{r} / B_{1}, \ldots, B_{N-r}\right)$ of $C_{N}$; it may therefore be infinitesimally displaced so as to induce the partition

$$
\left(A_{1}, \ldots, A_{r} / P, B_{1}, \ldots, B_{N-r}\right)
$$

of $C_{N+1}$, a partition induced by none of the planes of the set [ $\left.\pi\right]$. Thus $v_{k}(N)$ of the planar partitions of $C_{N}$ correspond to $2 v_{k}(N)$ distinct planar partitions of $C_{N+1}$. The remaining partitions of $C_{N}$ give rise to partitions of $C_{N+1}$, through the corresponding planes of the set $[\pi]$, that are a fortiori distinct from any of the $2 v_{k}(N)$ just considered. The latter are $v_{k}(N)-v_{k-1}(N)$ in number; hence there are at least

$$
v_{k}(N)+v_{k-1}(N)
$$

distinct partitions of $C_{N+1}$ by $(k-1)$-planes.

Conversely, any given planar partition of $C_{N+1}$ is similarly seen to be induced by one of the planes of the set $\left[\pi^{*}\right] \cup[\pi]$, so that there are just

$$
v_{k}(N)+v_{k-1}(N)
$$

distinct planar partitions of $C_{N+1}$. Thus $(C)$ is proved.

For $N-1 \geqq k$ the recurrence relation $(C)$ with the initial conditions $(B)$ now readily yields the result $(A)$ of the theorem. To deal with the case where $N-1<k$ we simply observe that the set of $N$ points lies in a $(N-1)$-plane imbedded in the $E_{k}$ so that the enumeration of planar partitions of this set of points in $E_{k}$ is equivalent to enumeration with $k=N-1$, to which the result just obtained applies. The theorem is now fully proved. 
3. The second question is answered by

Theorem 2. The number of partitions remaining when the partitioning plane is constrained to contain a given $j$-plane $(0 \leqq j \leqq k-1)$ is

$$
v_{k-j-1}(N)
$$

where $v_{i}(N)$ is given by equation $(A)$ above.

Proof. Let $E_{j}$ be the given $j$-plane (containing none of the $N$ given points), and let $E_{k-j}$ be any $(k-j)$-plane orthogonal to it, $E_{j}$ and $E_{k-j}$ having the single point $Q$ in common. Let the set $C_{N}$ of given points be projected orthogonally into $E_{k-j}$ to give a configuration $C_{N}^{\prime}$; and let $C_{N}^{\prime}$ be projected from $Q$ into a general $(k-j-1)$-plane in $E_{k-j}$ to give a configuration $C_{N}^{\prime \prime}$ of $N$ points in general position in that $(k-j-1)$-plane. Then there are as many partitions of $C_{N}$ into two groups by $(k-1)$-planes containing $E_{j}$ as there are partitions of $C_{N}^{\prime \prime}$ by unrestricted $(k-j-2)$-planes, namely

$$
v_{k-j-1}(N) \text {. }
$$

4. The relevance of these results for Cluster Analysis springs from the fact that certain criteria for cluster membership when individuals are represented as points in $E_{k}$ lead to the property that the convex hulls of different clusters do not intersect. It follows that a set of $N$ points in $k$ dimensions may be examined for partition into two clusters simply by evaluating the clustering criterion only for the $v_{k}(N)$ different planar partitions into two clusters when $N-1>k$, instead of the larger number $2^{N-1}$ of all possible associations of the $N$ points into two sets whose examination is normally necessary when the clustering criterion is not known to lead to such a property.

Neither the results nor the method of proof offer clues to practical algorisms for obtaining the set of all planar partitions without effectively considering en route the remainder of the $2^{N-1}$ associations into two sets. If this is not possible then the saving of effort implied by the theorem would be illusory unless the subsidiary calculations performed on each of the $2^{N-1}$ associations were much less involved than the evaluation of the clustering criterion. Since the latter is usually a simple function of the Euclidean distances between the points, such a comparison is unlikely to favour using the results given here. It is very likely that determining whether such algorisms exist would also yield a much better proof of Theorem 1 than the present somewhat cumbersome one.

5. Although it is interesting to speculate on the problem of the enumeration of the number of planar partitions of $N$ points in $k$ dimensions into two sets, one with $r$ points and the other with $N-r$, for given $r$, yet the observed fact that this number depends on the configuration of the points warns that it may be too complicated to be worth solving, despite the fact that there would be some interest in the answer from the point of view of practical Cluster Analysis. 
6. Some discussion of the problems and methods of Cluster Analysis will be found in Edwards and Cavalli-Sforza (6), with reference to a particular method of segregation into clusters there proposed.

The numbers $v_{k}(N)$ defined by equation $(A)$ have appeared before in the literature in connection with the partition of space into regions by planes. Carver (2), Blumenthal and Gillam (3), Buck (4), and Robinson (5), all consider such problems, and some give references to earlier work. Unfortunately their problems were not obviously equivalent to the ones considered here, nor were their methods of proof readily adaptable to the present case, though clearly there is actually an intimate connection between the problems. An early reference to these numbers is Schläfli $(\mathbf{1}) \dagger$ who finds the number of regions into which $E_{k}$ is partitioned by a set of $n$ hyperplanes to be $v_{k}(n+1)$, by establishing the same recurrence relation as $(C)$ of this paper. See also Winder (7).

\section{Acknowledgment}

The author is heavily indebted to Dr A. W. F. Edwards of Aberdeen University, for discussion of Cluster Analysis and of the questions raised in this paper, which greatly clarified the issues and suggested the result of Theorem 1.

\section{REFERENCES}

(1) L. SchläFLI, Theorie der vielfachen Kontinuität (Berne, 1852), Ges. Math. Abh. Vol. I, p. 209 (Basel, 1950).

(2) W. B. CARVER, The polygonal regions into which a plane is divided by $n$ straight lines. Amer. Math. Monthly, 48 (1941), 667-675.

(3) L. M. Blumenthal and B. E. Gillam, Distribution of points in $n$-space. Amer. Math. Monthly, 50 (1943), 181-185.

(4) R. C. Buck, Partition of space, Amer. Math. Monthly, 50 (1943), 541-544.

(5) H. A. Robinson, A problem of regions. Amer. Math. Monthly 52 (1945), 33-34.

(6) A. W. F. Edwards and L. L. Cavalli-Sforza, A method for Cluster Analysis, Biometrics, 21 (2) (1965), 362-375.

(7) R. O. WInder, Partitions of $N$-space by Hyperplanes, SIAM J. Appl. Math. 14 (4) (1966), 811-818.

DePartMENT OF STATISTICS

UNIVERSITY OF EDINBURGH

I I am indebted to the referee for this reference. 